\title{
Gewünschter Druckabfall von distal nach proximal bei allen Fabrikaten gewährleistet?
}

— Eine aktuelle biomechanische Untersuchung zeigte, dass nicht alle am Markt verfügbaren medizinischen Thromboseprophylaxestrümpfe (MTPS) den geforderten kontinuierlichen Druckabfall von distal nach proximal gleich gut gewährleisten.

Bei immobilen und bettlägerigen Patienten ist v.a. durch die fehlende Muskelaktivität ein wichtiger Mechanismus ausgeschaltet, der den venösen Rückstrom des Bluts zum Herzen fördert. MTPS schaffen hier einen Ausgleich: Sie üben Druck auf die oberflächlichen und tiefen Venen der Beine aus und verkleinern so den Gefäßquerschnitt. Dadurch fließt das Blut mit erhöhter Geschwindigkeit zum Herzen zurück, was der Entstehung von gefährlichen Blutgerinnseln entgegenwirkt. Zusätzlich wird der venöse Rückfluss durch ein abgestuftes (graduiertes) Druckprofil, das durch die Dehnung des Gestricks entsteht, positiv beeinflusst.

Der Andruck der Strümpfe im Bereich der Fessel sollte nach bisherigen Empfehlungen rund $18 \mathrm{mmHg}$ betragen und auf etwa $8 \mathrm{mmHg}$ am Oberschenkel abfallen. Unter diesen Druckverhältnissen besteht i. d. R. keine Gefahr für eine arterielle Unterversorgung der Extremitäten oder für einen verminderten venösen Rückstrom.

Dass nicht alle MTPS die geforderten biomechanischen Eigenschaften aufweisen, hat die aktuelle Untersuchung verschiedener Strumpffabrikate deutlich gemacht, die Prof. Marc Kraft, Berlin, durchführte. Damit wurde eine erste Forderung der im letzten Jahr neu gegründeten Expertengruppe "Thromboseprophylaxe" erfüllt. Diese hatte vermutet, dass sich MTPS im Hinblick auf das geforderte graduierte
Druckprofil deutlich voneinander unterscheiden, und dass sich die mit einem bestimmten Strumpftyp gewonnenen Studienergebnisse nicht ohne weiteres auf andere Fabrikate übertragen lassen.

„Die Ergebnisse der Testung zeigen nun, dass wir mit dieser Annahme richtig gelegen haben", sagte Prof. Peter Kujath, Lübeck. Um valide Aussagen zum Effekt von MTPS zusätzlich zu einer medikamentösen Thromboseprophylaxe machen zu können, seien neue klinische Studien mit biomechanisch exakt charakterisierten Strümpfen zu fordern. „Ohne eine bessere Datenlage ist die seriöse Neubewertung des Nutzens bzw. Zusatznutzens von MTPS nicht möglich", bekräftigte auch Dr. Colin M. Krüger, Berlin.

- Red.

Quelle: MDI Starnberg

\section{Herzneurose}

\section{Pflanzliches Kombipräparat stabilisiert den Herzrhythmus}

- Auch wenn beim Herzneurotiker psychosomatische Anteile zugrundeliegen können, bedarf er einer adäquaten Behandlung, denn seine Ängste und Beschwerden sind real. Ein rhythmusstärkendes pflanzliches Kombinationspräparat bietet natürliche Hilfe an.

Mit dem Widerspruch zwischen starken Beschwerden und Messwerten im Normbereich fühlen sich Patienten mit funktionellen Herzbeschwerden oft allein gelassen. Immerhin leiden in Deutschland 2-5\% der Bevölkerung an Herzneurosen ( 1,6 bis 4 Millionen Menschen). Männer sind doppelt so häufig wie Frauen betroffen.

Für betroffene Patienten ist es schwer, funktionelle von organischen Herzbeschwerden zu unterscheiden. Symptome wie funktionelle Dyskardien mit Unruhe, Herzklopfen und Herzschmerzen können Angstzustände auslösen, auch wenn keine herzorganischen Ursachen zu finden sind.

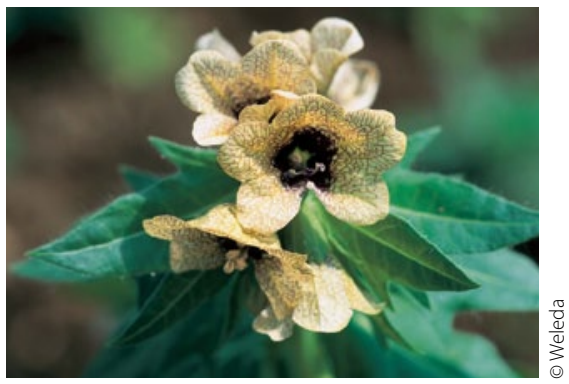

Bilsenkraut und Eselsdistel sorgen jeweils für die Stärkung systolischer Funktionen, die Kräftigung diastolischer Prozesse und die Stabilisierung der rhythmischen Abläufe des Herzens. Kombiniert haben die Substanzen eine regulative Wirkung auf das Herz-Kreislauf-System und lindern die subjektiven Beschwerden.

Wegen seiner guten Verträglichkeit und Das Phytotherapeutikum enthält verdünnte Bilsenkraut-Tinktur und ist verschreibungspflichtig.

„In solchen Fällen lässt sich oft eine fehlende Frequenzvariabilität in Ruhe feststellen“, weiß Dr. Adelheid Grünewald-Fritsch, Stuttgart, aus Erfahrung. Diese mangelhafte Anpassungsfähigkeit des Herzens an Belastung und Ruhe ließe sich durch ein anthroposophisches Kompositionsmittel (Cardiodoron ${ }^{\oplus}$ ) verbessern. Bestandteile aus den drei Heilpflanzen Schlüsselblume, fehlender Wechselwirkungen kann das auch in Kombination mit schulmedizinischen Präparaten eingesetzt werden. Typische GKV-erstattungsfähige Anwendungen sind funktionelle Herz-KreislaufBeschwerden, Rhythmusstörrungen, akute Kreislaufbelastungen, arterielle Hypertonie und Basistherapie bei Herzinsuffizienz, nicht jedoch Hypotonie.

- Dr. Ulrike Fortmüller

Quelle: „Cardiodoron ${ }^{\oplus}$-Presse-Round-Table“, München, April 2012 (Veranstalter:Weleda) Medikament sowohl als Monotherapie als 